\title{
Structure-function relations in phosphorylcholine-binding mouse myeloma proteins
}

\author{
(nuclear magnetic resonance/binding subsites/hapten environment)
}

\author{
ANDREW M. GOETZE AND JOHN H. RICHARDS \\ Church Laboratory of Chemical Biology, Division of Chemistry and Chemical Engineering, California Institute of Technology, Pasadena, California 91125
}

\begin{abstract}
The binding site interactions between the phosphorylcholine (phosphocholine)-binding mouse myeloma proteins TEPC 15, W3207, McPC 603, MOPC 167, and MOPC 511 and the isotopically substituted hapten phosphoryl[methyl-13 $\mathrm{C}$ ]choline have been investigated using ${ }^{3} \mathrm{C}$ and ${ }^{31} \mathbf{P}$ nuclear magnetic resonance (NMR) spectroscopy. Each protein exhibits a unique NMR pattern, but extensive similarities in chemical shift parameters upon binding of hapten to immunoglobulin suggest a significant degree of conservation of important hapten-binding site interactions. Moreover, independent binding studies, in conjunction with the NMR data, allow construction of a simple model of the binding sites of these antibodies, analyzed in terms of the relative strength of interaction between hapten and two main subsites. The NMR evidence supports the view that the heavy chains of these proteins dominate in interacting with bound phosphorylcholine; the various subspecificities of these proteins for phosphorylcholine analogues can be accounted for by amino acid changes in the hypervariable regions of the heavy chains.
\end{abstract}

The heavy and light chains of an antibody cooperate to form the specificity site for recognition of a particular antigen. This work is aimed at exploring the degree to which these two chains determine specificity and the effect of small structural changes in the antibody on the molecular aspects of its interaction with antigens.

A number of myeloma proteins produced by plasmacytomas of BALB/c mice have been found to interact specifically with phosphorylcholine $(P$-Cho; the name phosphocholine is recommended by the International Union of Pure and Applied Chemistry) (1-3) and sequence analysis of the heavy chains of several of these proteins $(4,5)$ has revealed a degree of homology greater than $85 \%$. Though still incomplete, the sequence data on the light chains suggest a division into at least three subsets with sequences differing widely between members of different subsets (6-8). Though these antibodies have similar affinities for $\boldsymbol{P}$-Cho, they exhibit different affinities for its structural analogues (9). Because of the extensive homology of the heavy chains in these proteins and the correlation between the subspecificity of an individual protein and the subgroup of the light chain, the heavy chain may well be the principal determinant of the specificity for $P$-Cho while the light chain "fine tunes" the subspecificity (8).

The antibodies studied to date show a high degree of conservation of their tertiary and quaternary structures. Thus, one can construct plausible three-dimensional binding sites using sequence data together with structural information obtained by $x$-ray diffraction studies of other, related immunoglobulins. For example, the three-dimensional structure of the Fab' fragment of $\mathrm{M} 603$, a typical mouse myeloma protein that binds $P$-Cho, has been determined to 3.1 A resolution (10). This in-

Abbreviations: NMR, nuclear magnetic resonance; $P$-Cho, phosphorylcholine; mouse plasmacytomas: T15, TEPC 15; M603, McPC 603; M167, MOPC 167; M511, MOPC 511. formation, together with the existence of a well-characterized group of similar immunoglobulins, has afforded the opportunity to study the molecular details of structure-function relationships and, in particular, to assess the importance of individual chains and amino acid residues to the interactions between antibody and antigen (11). Such studies should help to reveal the molecular source of the exquisite discriminatory powers exhibited by the immune response.

We have previously discussed the use of ${ }^{31} \mathrm{P}$ and ${ }^{13} \mathrm{C}$ magnetic resonance techniques to study the binding of $P$-Cho to M603 (12) and now extend this type of study to four other myeloma proteins that specifically bind $P$-Cho. In this report we emphasize differences in interaction between antibody and hapten caused by changes of amino acids in the subregions of the antibody binding site.

\section{MATERIALS AND METHODS}

Plasmacytomas TEPC 15 (abbreviated T15), McPC 603 (M603), and W3207 were obtained from the Salk Institute, San Diego, CA; MOPC 167 (M167) and MOPC 511 (M511), through the courtesy of Dr. Leroy Hood. $\left[\right.$ methyl- $\left.{ }^{14} \mathrm{C}\right]$ Choline chloride was purchased from New England Nuclear and L$\alpha$-glycerophosphorylcholine (95\%) from Sigma. Phosphonocholine was a gift from Dr. Leroy Hood. Purification of protein and the experimental details have been described elsewhere (12).

The numbering system of heavy chain amino acids is that of Rudikoff and Potter for M603 (13).

\section{RESULTS}

Table 1 summarizes data obtained by equilibrium dialysis on the thermodynamics of binding by five myeloma proteins together with background information. Our binding constants $\left(K_{\mathrm{a}} \mathrm{s}\right)$ for $P$-Cho agree reasonably well with published results $(3,14-16)$. The binding constant for W3207 is larger than for any other myeloma protein studied to date. As previously suggested (1), M511 shows a relatively high affinity for choline.

Table 2 summarizes ${ }^{31} \mathrm{P}$ and ${ }^{13} \mathrm{C}$ nuclear magnetic resonance (NMR) results of interactions between the various proteins and phosphoryl $\left[\right.$ methyl $\left.{ }^{13} \mathrm{C}\right]$ choline. Binding of hapten to antibody generally causes a $1.2-1.3 \mathrm{ppm}$ upfield shift of the ${ }^{13} \mathrm{C}$ methyl resonance relative to its position when free in solution; M603 provides the exception with a smaller upfield shift of $0.7 \mathrm{ppm}$. The ${ }^{31} \mathrm{P}$ resonance moves $1.5 \mathrm{ppm}$ upfield on binding for all of the proteins with the exception of M167, for which the chemical shift for the bound form is the same as for the hapten free in solution.

Because the value of the dissociation rate constant of the antibody-hapten complex, $k_{\text {off }}$, is similar to the change in chemical shift occasioned by binding of hapten to antibody, 
Table 1. Properties of phosphorylcholine-specific myeloma proteins

\begin{tabular}{|c|c|c|c|c|c|c|}
\hline \multirow[b]{2}{*}{ Property } & \multicolumn{5}{|c|}{ Myeloma protein } & \multirow[b]{2}{*}{ Ref. } \\
\hline & $\mathrm{T} 15$ & W3207 & M603 & M167 & M511 & \\
\hline Idiotype & A & $?(\neq \mathrm{A})$ & B & C & D & 1,20 \\
\hline Light chain subgroup & I & II & II & III & III & $5-7$ \\
\hline $\begin{array}{l}\text { Number of heavy chain } \\
\text { differences from T15 }\end{array}$ & 0 & 7 & 5 & 13 & 5 & 5 \\
\hline $\begin{array}{l}\text { Phosphorylcholine } K_{\mathrm{a}} \times 10^{-5}, \mathrm{M}^{-1} \\
4^{\circ} \\
30^{\circ}\end{array}$ & $\begin{array}{c}17.5 \\
3.5\end{array}$ & $\begin{array}{c}30.4 \\
7.0\end{array}$ & $\begin{array}{l}8.2 \\
1.0\end{array}$ & $\begin{array}{l}7.4 \\
1.2\end{array}$ & $\begin{array}{l}0.43 \\
0.15\end{array}$ & \\
\hline $\begin{array}{l}\text { Subspecificity* } \\
\text { Choline } \\
\text { L- } \alpha \text {-Glycerophosphorylcholine } \\
\text { Phosphonocholine }\end{array}$ & $\begin{array}{l}550 \\
11 \\
95\end{array}$ & $\begin{array}{c}600 \\
4 \\
15\end{array}$ & $\begin{array}{l}870 \\
10 \\
11\end{array}$ & $\begin{array}{l}10 \\
0.8 \\
33\end{array}$ & $\begin{array}{c}3 \\
0.7 \\
-\end{array}$ & \\
\hline
\end{tabular}

* Represented as $K_{\mathrm{a}}$ (phosphorylcholine) $K_{\mathrm{a}}$ (inhibitor). Data for W3207 and M511 were determined by direct equilibrium dialysis (choline) or inhibition of phosphorylcholine binding (L- $\alpha$-glycerophosphorylcholine and phosphonocholine) at $4^{\circ}$. Data for T15, M603, and M167 are taken from Leon and Young (9).

exchange-broadened resonances were observed in all cases but with differences characteristic of each protein. For example, binding of excess $P$-Cho to W3207, M603, and M167 resulted in separate peaks corresponding to free and bound hapten for both the ${ }^{13} \mathrm{C}$ and ${ }^{31} \mathrm{P}$ signals, whereas binding to M511 resulted in a single resonance corresponding to the weighted average of the two environments. Binding of $P$-Cho to T15 gave two separate ${ }^{31} \mathrm{P}$ signals but only a single greatly broadened ${ }^{13} \mathrm{C}$ resonance. Table 2 also collects the values of $k_{\text {off }}$, estimated from the observed change in chemical shift on binding and the extent of exchange broadening, as well as the calculated values of the rate constant for association $\left(k_{\text {on }}=K_{\mathrm{a}} \cdot k_{\text {off }}\right)$. Except for $\mathrm{M} 603$, the rate constants from the ${ }^{13} \mathrm{C}$ and ${ }^{31} \mathrm{P}$ observations are in good agreement.

\section{DISCUSSION}

Subsite Interactions among PhosphorylcholineBinding Myeloma Proteins. Particular regions of the binding site responsible for a certain type of interaction (e.g., electrostatic, hydrogen bonding, or hydrophobic) with a portion of the bound ligand have been termed interaction subsites; their existence was postulated by Haselkorn et al. (17) as a result of kinetic experiments on a myeloma protein that binds dinitrophenyl derivatives. In this model, the total binding energy represents an appropriate summation of the contributions from each individual subsite-ligand interaction. This serves as a convenient basis for our discussion of the binding of $\boldsymbol{P}$-Cho or its analogues to myeloma proteins with specificity for $P$ Cho.

For binding of $P$-Cho one may envision three subsites as contributing substantially to hapten binding: a negatively charged subsite for interaction with the positive quaternary nitrogen; a positive, possibly hydrogen-bond-donating subsite for interaction with the dianionic phosphate; and a hydrophobic subsite for interaction with the two central methylene groups of $P$-Cho. Specific electrostatic interactions, consistent with the first two of these postulated subsites, have been observed in the three-dimensional structure of the binding site of M603 which was determined by $x$-ray diffraction (10).

For consideration of the phosphate-binding subsite, proteins T15, W3207, and M603 are useful. These all cause identical hapten ${ }^{31} \mathrm{P}$ shifts and show similar $k_{\text {off }}$ rates calculated from their ${ }^{31} \mathrm{P}$ spectra, suggesting similar subsite interactions with the phosphate group. Their affinity for choline is lower by a factor of $600-900$ than that for $P$-Cho, as would be anticipated if interactions with the dianionic phosphate group are important for binding. Also, their affinity for $\mathrm{L}-\alpha$-glycerophosphorylcholine is lower by a factor of 4-10 compared to $P$-Cho, suggesting steric interference and/or loss of electrostatic binding interaction when the larger, monoanionic L- $\alpha$-glycerophosphate group binds in the phosphate pocket. These results indicate that, for these three proteins, the phosphate subsites are probably very similar; their different absolute affinities for each of $P$ Cho, choline, and L- $\alpha$-glycerophosphorylcholine arise from differing interactions with the trimethylammonium region of these ligands. The ${ }^{13} \mathrm{C}$ NMR results support this view and imply that M603 interacts differently than do T15 and W3207 with the methyl groups of the hapten. In addition, different $k_{\text {off }}$ rates were calculated from the ${ }^{13} \mathrm{C}$ spectra of labeled hapten interacting with these three proteins. Though M603 binds $P$-Cho less strongly than do T15 and W3207, M603 nevertheless has

Table 2. Phosphorylcholine chemical shifts and kinetic rate constants obtained with phosphorylcholine-specific myeloma proteins

\begin{tabular}{|c|c|c|c|c|c|}
\hline \multirow[b]{2}{*}{ Property } & \multicolumn{5}{|c|}{ Myeloma protein } \\
\hline & $\mathrm{T} 15$ & W3207 & M603 & M167 & M511 \\
\hline${ }^{13} \mathrm{C}$ shift on binding, ppm & 1.3 & 1.2 & 0.7 & 1.3 & 1.2 \\
\hline${ }^{31} \mathrm{P}$ shift on binding, ppm & 1.5 & 1.5 & 1.5 & 0 & 1.5 \\
\hline$k_{\text {off }}$ from ${ }^{13} \mathrm{C}$ spectra, sec ${ }^{-1}$ & 107 & 34 & 10 & 53 & 195 \\
\hline$k_{\text {off from }}{ }^{31} \mathrm{P}$ spectra, sec $^{-1}$ & 63 & 47 & 38 & - & 189 \\
\hline$k_{\text {on }} \times 10^{-6}$ from ${ }^{13} \mathrm{C}$ spectra, $\mathrm{M}^{-1} \mathrm{sec}^{-1}$ & 37 & 24 & 0.94 & 6.4 & 2.9 \\
\hline$k_{\text {on }} \times 10^{-6}$ from ${ }^{31} \mathrm{P}$ spectra, $\mathrm{M}^{-1} \mathrm{sec}^{-1}$ & 22 & 33 & 3.8 & - & 2.8 \\
\hline
\end{tabular}


Table 3. Postulated strength of subsite interactions of phosphorylcholine-specific myeloma proteins

\begin{tabular}{ccccccc}
\hline & \multicolumn{5}{c}{ Myeloma protein } \\
\cline { 2 - 7 } & T15 & W3207 & M603 & M167 & M511 \\
\hline $\begin{array}{c}\text { Choline subsite } \\
\text { Phosphate subsite } \\
\text { Total }\end{array}$ & $\begin{array}{c}++ \\
++\end{array}$ & +++ & ++ & ++ & +++ & + \\
$K_{\mathrm{a} \times 10^{-5}} \times \mathrm{M}^{-1}$ & $6+$ & $\frac{++}{4+}$ & $\frac{+}{4+}$ & + \\
$\left(30^{\circ}\right)$
\end{tabular}

+ , weak; ++ , moderate; +++ , strong

a lower value of $k_{\text {off, }}$ which requires that it have a substantially lower association rate constant than do T15 and W3207.

Comparative affinities do not, however, always correlate with subsite interactions, as is demonstrated by comparison of binding by M167 and M511, both of which have affinities for choline comparable to those for $P$-Cho although they have quite different binding site interactions. For example, M167 is unique among the proteins studied in causing no shift of the ${ }^{31} \mathrm{P}$ hapten resonance on binding, suggesting a very different subsite for binding the phosphate group (see below). The choline subsite of M167 appears, however, to be very similar to the subsites of $\mathrm{T} 15$ and $\mathrm{W} 3207$. Though the hapten ${ }^{13} \mathrm{C}$ and ${ }^{31} \mathrm{P}$ shifts induced by binding to M511 are both similar to those seen on binding to T15 and W3207 (indicating similar interactions with the phosphate and trimethylammonium groups of the hapten), the dissociation rate constant of the M511-phosphorylcholine complex is an order of magnitude higher than that of the other proteins and is reflected in a decreased binding constant. This unexpectedly low affinity cannot be traced to noticeably different interactions between antibody and trimethylammonium or phosphate group, but appears due to a generally poorer complementarity between hapten and binding site.

Table 3 summarizes the postulated subsite interactions deducible from the NMR and binding data. One sees that affinities for $P$-Cho and related haptens can be modulated by structural changes in relatively independent subregions of the binding pockets of these antibodies, which are known to exhibit diverse specificities for a variety of bacterial antigens $(3,18)$, a diversity which likely arises from these differences in subsites.

Comparison of the dynamic aspects of hapten binding by these five proteins shows that differences in affinity result not only from differing dissociation constants reflecting, probably, differences in complementarity between antibody and hapten, but also from differing association rate constants. The calculated association rate constants are typical of those previously observed for other antibody-hapten systems $(17,19-21)$ and in particular, the $k_{\text {on }}$ value of $4.6 \times 10^{7} \mathrm{M}^{-1} \mathrm{sec}^{-1}$ calculated by Pecht (19) for HOPC 8 agrees with our estimates of 2 to $4 \times 10^{7}$ $\mathrm{M}^{-1} \mathrm{sec}^{-1}$ for T15, a protein idiotypically identical to HOPC 8. However, the association rate constants for M603, M167, and M511 are lower by an order of magnitude than those for T15 and W3207, indicating that T15 and W3207 allow more ready access of hapten to the binding pocket than do the other three proteins.

Relation between Differences in Binding Sites and Three-Dimensional Structure. The similarity of both the ${ }^{13} \mathrm{C}$ and ${ }^{31} \mathrm{P}$ hapten chemical shifts among most of the proteins examined suggests a high degree of similarity in the important electrostatic interactions between hapten and binding site. M167, however, shows an unusual phosphate-binding subsite and M603 a unique choline subsite; we shall examine the possible molecular origins for these anomalies.

The heavy chain seems to play the dominant role in the interaction between these antibodies and hapten (6). This is supported by the high degree of conservation of heavy chain sequence among the $P$-Cho-binding immunoglobulins and also by the presence of three widely differing light chain sequences among the five proteins. Such a view is confirmed by the three-dimensional structure of the M603-P-Cho complex, in which the phosphate-binding subsite is found to be formed exclusively by heavy chain residues with the following amino acids being in direct contact with $P$-Cho: Tyr $33(\mathrm{H})$, Glu $35(\mathrm{H})$, $\operatorname{Arg} 52(\mathrm{H})$, Glu 59(H), Trp 104a $(\mathrm{H})$, and an unidentified residue at position 96 of the light chain $(10,11)$. The first five of these residues, as well as the ionically important Lys $54(\mathrm{H})$, are conserved among most of the $P$-Cho-binding mouse myeloma proteins.

We have previously interpreted the ${ }^{31} \mathrm{P}$ chemical shifts on binding of P-Cho (+1.5 ppm) as being caused largely by hydrogen bonding between specific amino acid residues and the phosphate group (12). Ml67 shows no such shift. Why? This does not necessarily correlate with the relatively high affinity of M167 for choline because M511 also has this high choline affinity but nevertheless causes a $+1.5 \mathrm{ppm}$ shift for bound $P$-Cho. We suggest that absence of a $+1.5 \mathrm{ppm}$ shift on binding to $\mathrm{Ml} 67$ results from presence in the phosphate subsite of an additional cationic residue, $\operatorname{Arg} 58 \mathrm{a}(\mathrm{H})$, which is unique to M167. Examination of the M603 x-ray structure reveals that heavy chain hypervariable region 2 (residues 50-65) forms a crucial portion of the phosphate-binding subsite. Relative to T15, M167 has three amino acid substitutions in this second hypervariable region (Asn $53 \rightarrow$ Ser, Asn $56 \rightarrow$ His, and Thr $58 \mathrm{a} \rightarrow \mathrm{Arg}$ ). Addition of a third cationic residue (Arg 58a) (in addition to Arg 52 and Lys 54, both of which stablize the bound phosphate) could cause a diamagnetic downfield effect that offsets the upfield shift resulting from the usual hydrogen bond formation.

Additional evidence for a strong interaction of the phosphate subsite of M167 with the hapten comes from the increased ${ }^{31} \mathrm{P}$ linewidth of the bound hapten $\left(\Delta v_{1 / 2}=24 \mathrm{~Hz}\right)$, which suggests immobilization of the phosphate group of the bound hapten as would result from formation of hydrogen bonds.

The anomalous ${ }^{13} \mathrm{C}$ shift observed for M603 is more difficult to rationalize because the light chain contributes to formation of the choline subsite and sequence data for the light chains are still incomplete. However, the other four proteins have, relative to $\mathrm{M} 603$, an extra residue in the third hypervariable region of the heavy chain [residue $103 \mathrm{a}(\mathrm{H})$ ], and such an insertion could have a significant effect on the binding interaction in this subsite.

Importance of Specific Chains in the Formation of a Functional Binding Site. We would like to suggest that, to a first approximation, the various subspecificities in these proteins result from the independent modification of one of the two main subsites through changes in critical heavy chain hypervariable residues. Such changes require the concomitant presence of a light chain that is compatible with such a specifically altered heavy chain. This would explain the low $P$-Cho affinity of heterologous hybrids of S107 and M511 (22) as well as our observations indicating that both heterologous hybrids of T15 and Ml67 have affinities for $P$-Cho of less than $10^{4} \mathrm{M}^{-1}$. Thus, though it may not extensively interact directly with the hapten, the light chain plays a critical role in determining antibody specificity by serving as a precisely complementary structure with which the heavy chain can join in a mutual embrace to 
create the binding site. Padlan et al. (11) have pointed out that the extensive contact between light chain hypervariable region 1 and heavy chain hypervariable region 3 in M603 could be an example of one way in which highly specific light-heavy chain pairing is achieved in $P$-Cho-binding proteins.

The tendency for proteins containing the same type light chain to share similar subspecificities even though dissimilar environments for bound $P$-Cho are observed by NMR allows the possibility that the light chain, though largely uninvolved in contacting the important regions of the ligand, nevertheless indirectly affects ligand binding properties through synergistic interactions with the heavy chain.

Comparison of Phosphorylcholine-Binding Sites. We may now summarize our understanding of the individual binding sites of these proteins. W3207, which has the highest affinity for $P$-Cho, forms strong interactions with both ends of the hapten. T15 has differences from W3207 in the third hypervariable region of its heavy chain which, by analogy to the $x$-ray structure of M603, would be expected to influence mainly the subsite for choline. Accordingly, T15 exhibits slightly weaker interactions in this subsite, but retains the strong phosphate subsite interactions of W3207; the net result is a 2-fold decrease in the affinity of T15 for $P$-Cho. M603 retains the W3207 type light chain but has significant differences throughout the heavy chain, causing a substantial alteration in the choline subsite; the overall affinity of M603 for $P$-Cho is reduced by a factor of 4-6 relative to that of W3207. M167 has a heavy chain with a wider diversity from the W3207 prototype than that of any of the other proteins. M167 also has a different type of light chain. These changes give rise to a greatly altered phosphate subsite, where decreased specificity is attested by the high relative affinity for choline and L- $\alpha$-glycerophosphorylcholine. M511 has a light chain of the M167 type and only a moderate number of amino acid differences in its heavy chain relative to W3207; we have no explanation for the noticeably decreased affinity of M511 for P-Cho.

\section{CONCLUSIONS}

The generally similar hapten-binding site interactions observed for this series of five idiotypically distinct mouse myeloma proteins demonstrates a general conservation of at least the important electrostatic interactions in the binding sites of this family of immunoglobulins. This conservation is, however, not absolute, and deviations are observed in subregions of the binding pockets of M167 and M603. The hapten-binding site interactions, as monitored by the ${ }^{13} \mathrm{C}$ and ${ }^{31} \mathrm{P}$ nuclei of the $P$-Cho hapten, correlate with the binding affinities of these proteins for various haptens. The concept of subsite interactions has proved especially fruitful in understanding these effects.

Among these proteins, changes in binding properties can be accounted for largely by differences in the heavy chains, which appear to play the dominant role in determining specificity for
$P$-Cho. Though the fundamental significance of the pairing of similar heavy chains with at least three distinct types of light chains remains obscure, hybridization experiments suggest that high affinity for $\boldsymbol{P}$-Cho requires specific association between heavy and light chains.

This is Contribution 5452 from the Division of Chemistry and Chemical Engineering, California Institute of Technology.

The costs of publication of this article were defrayed in part by the payment of page charges from funds made available to support the research which is the subject of the article. This article must therefore be hereby marked "advertisement" in accordance with $18 \mathrm{U}$. S. C. $\$ 1734$ solely to indicate this fact.

1. Potter, M. \& Lieberman, R. (1970) J. Exp. Med. 132, 737751.

2. Leon, M. A. \& Young, N. M. (1970) Fed. Proc. 29, 437.

3. Potter, M. (1972) Physiol. Rev. 52, 631-719.

4. Hood, L., Campbell, J. H. \& Elgin, S. C. R. (1975) Annu. Rev. Genet. 9, 305-353.

5. Hood, L., Loh, E., Hubert, J., Barstad, P., Eaton, B., Early, P., Furhman, J., Johnson, N., Kronenberg, M. \& Schilling, J. (1976) Cold Spring Harbor Symp. Quant. Biol., in press.

6. Barstad, P., Rudikoff, S., Potter, M., Cohn, M., Konigsberg, W. \& Hood, L. (1974) Science 183, 962-964.

7. Claflin, J. L., Rudikoff, S., Potter, M. \& Davie, J. M. (1975) J. Exp. Med. 141, 608-619.

8. Barstad, P. (1975) Ph.D. Dissertation, California Institute of Technology, Pasadena, CA.

9. Leon, M. A. \& Young, N. M. (1971) Biochemistry 10, 14241429.

10. Segal, D. M., Padlan, E. A., Cohen, G. H., Rudikoff, S., Potter, M. \& Davies, D. R. (1974) Proc. Natl. Acad. Sci. USA 71, 4298-4302.

11. Padlan, E. A., Davies, D. R., Rudikoff, S. \& Potter, M. (1976) Immunochemistry 13, 945-949.

12. Goetze, A. M. \& Richards, J. H. (1977) Biochemistry, 16, 228232.

13. Rudikoff, S. \& Potter, M. (1974) Biochemistry 13, 4033-4038.

14. Metzger, H., Chesebro, B., Hadler, N. M., Lea, J. \& Otchin, N. (1971) Progress in Immunology (Academic Press, New York \& London), pp. 253-267.

15. Pollet, R. \& Edelhoch, H. (1973) J. Biol. Chem. 248, 54435447.

16. Chesebro, B. \& Metzger, H. (1972) Biochemistry 11, 766-771.

17. Haselkorn, D., Friedman, S., Givol, D. \& Pecht, I. (1974) Biochemistry 13, 2210-2222.

18. Potter, M. \& Leon, M. A. (1968) Science 162, 369-371.

19. Pecht, I. (1974) in The Immune System: Genes, Receptors, Signals, eds. Sercarz, E. E., Williamson, A. R. \& Fox, C. F. (Academic Press, New York), pp. 15-35.

20. Sehon, A. H. (1963) Ann. N.Y. Acad. Sci. 103, 626-629.

21. Kelly, K. A., Sehon, A. H. \& Froese, A. (1971) Immunochemistry 8,613-625.

22. Sher, A., Lord, E. \& Cohn, M. (1971) J. Immunol. 107, 12261234. 